\title{
Using "Integrated Reminders of Nursing Written Assignment" to Reduce the Interruptions in Nursing Care
}

\author{
Chia-Hui CHU ${ }^{\mathrm{a}, 1}$ and Shu-Hui WENG ${ }^{\mathrm{a}}$

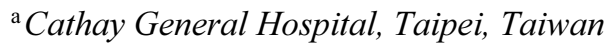

\begin{abstract}
Nursing interruptions can suspend the nursing process and distracting the nurses' attention. In a nursing information system, there are lots of pop-up windows to notify the nurses to complete the nursing records. These notifications can also interrupt the original work. We proposed an "Integrated Reminders of Nursing Written Assignment" system to reduce the number of interruptions from the nursing information system. The result showed that the number of pop-up windows decreased from 489.48 to 0 per shift. The completeness of the nursing records raised from $97.3 \%$ to $100 \%$. The "Integrated Reminders of Nursing Written Assignment" can effectively reduce the frequency of nursing interruptions and improve the quality of nursing records.
\end{abstract}

Keywords. Nursing interruptions, integrated reminders, completeness of nursing written assignment

\section{Introduction}

Nursing interruptions are the sudden events that interrupt or delay nursing workflow. When a nursing interruption occurs, the nursing process is suspended and the nurses' attention is distracted. Nursing interruptions also affect the efficiency and quality of clinical work, and is likely to cause adverse events. In addition, an interruption over 5 minutes can delay at least 23 minutes to the previous work [1][2]. In a nursing information system, there are lots of pop-up windows to notify the nurses to complete the nursing records. However, it also interrupts the nurses' original work. These abundant pop-up windows may lead to feelings of fatigue so that the important information is ignored by the nurses. Therefore, we proposed an "Integrated Reminders of Nursing Written Assignment" to reduce the interruptions in the nursing workflow.

\section{Methods}

First, to design the "Integrated Reminders of Nursing Written Assignment" system, we collected 15,546 nursing written assignments within a month. There were 17 kinds of nursing written assignment in our system, including six kinds of assessments when patients admitted, two kinds of bundle care note, fall risk assessment tool, pressure ulcer

${ }^{1}$ Corresponding Author, Chia-Hui Chu, Nursing Department, Cathay General Hospital, 280 Renai Rd. Sec.4, Taipei, Taiwan; E-mail: octopus@cgh.org.tw. 
scale, patient discharge screening tool, physical restraint assessment scale, nursing note, patient health problem list, close observation of patient vital signs sheets, intake and output sheet and pain scale. The completeness of nursing written assignments collected were $97.30 \%(15546 / 15978)$.

Second, we analyzed the types of pop-up windows in our nursing system. There were seven kinds of pop-up windows in our system (Figure 1).

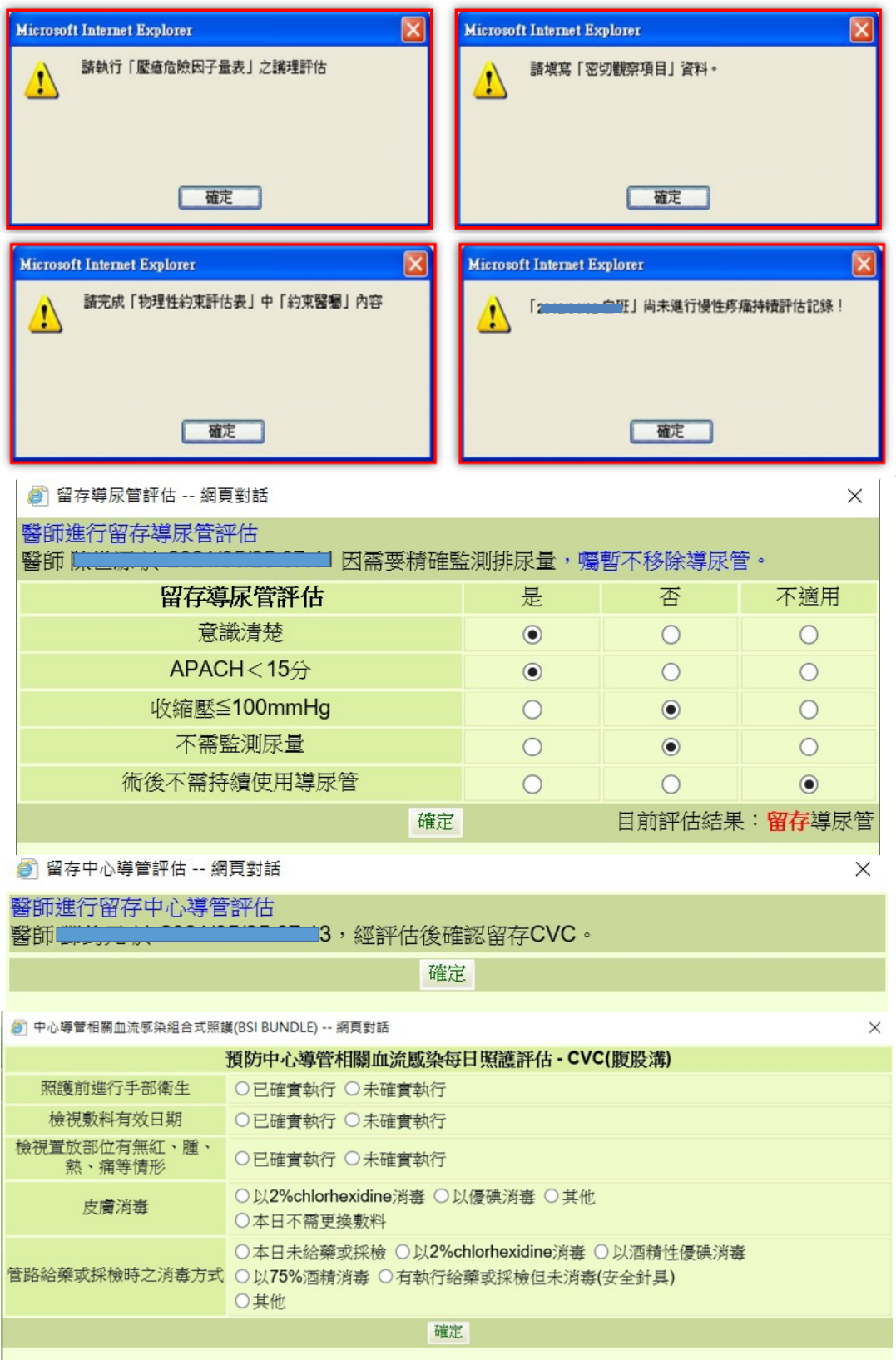

Figure 1. Seven kinds of pop-up windows in our system. 
Third, we collected the number of interruptions by examining the log file in our system. The log file recorded the events of the pop-up windows occurred and the windows were closed.

Fourth, we investigated the nurses' nursing written assignments in a shift. The average of the nursing written assignments was 3.42. In the original nursing information system, in order to avoid the incomplete of nursing written assignment, pop-up windows were displayed to remind the nurses to finish the nursing written assignments. But the nurses were tied up in their work, they chose to close the pop-up window. The average number of this kind of nursing interruptions in a shift was 489.48. It meant that the nurses spent 143 times to do the extra work.

In order to reduce interruptions, we designed the integrated reminders of nursing written assignment. We analyzed 7 kinds of pop-up windows, and redesigned the types of reminders. Like the social media reminders, we put the reminders on the top-right corner of the screen (Figure 2) and the nurses' working list (Figure 3).

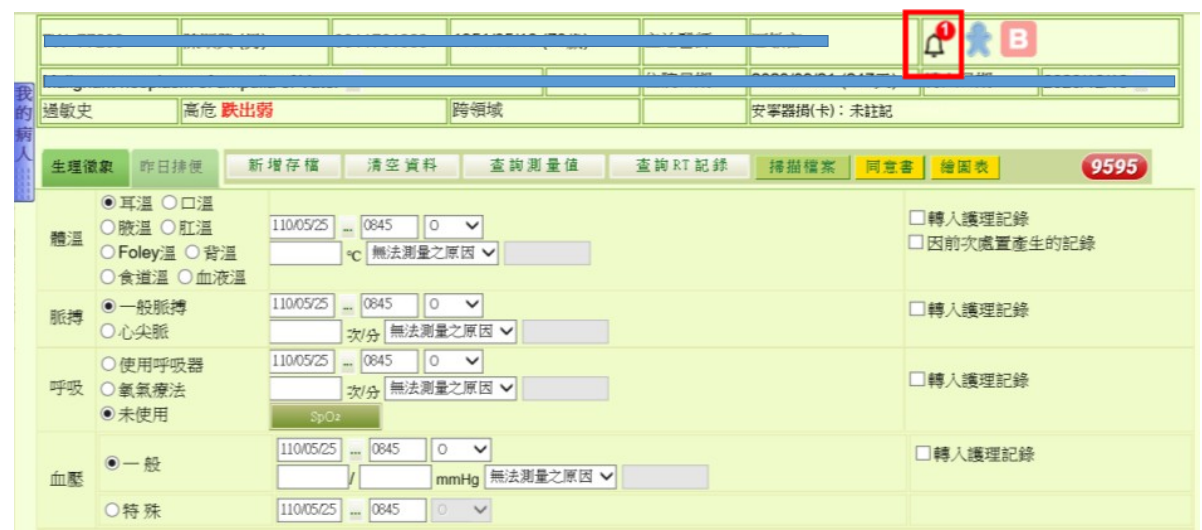

Figure 2. Reminders on the top-right corner of the screen.

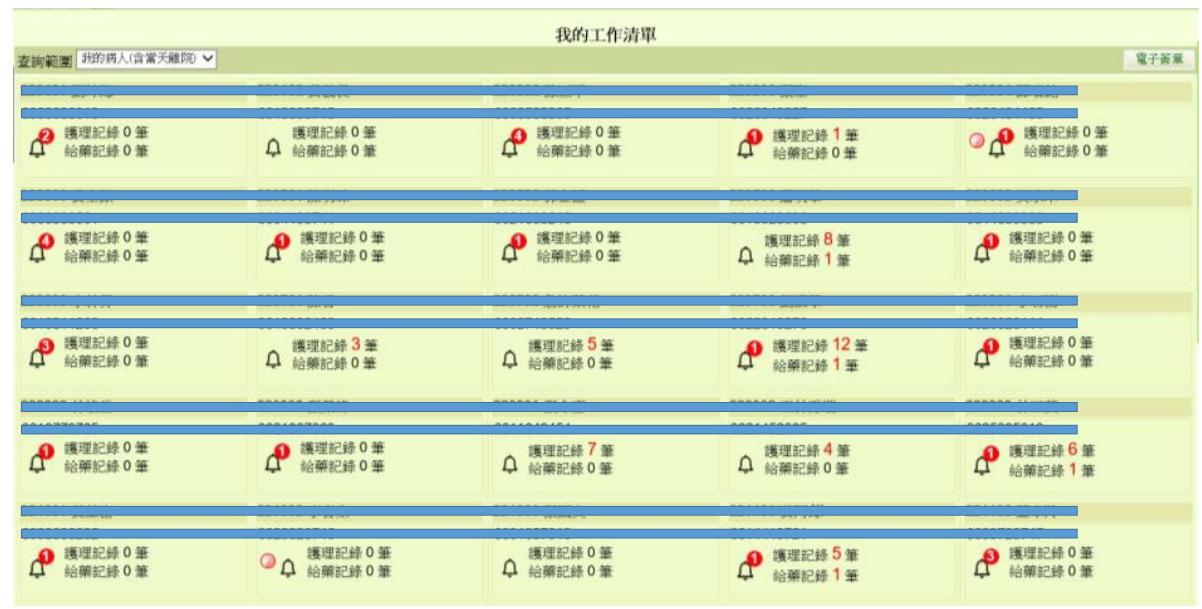

Figure 3. Reminders on the nurses' working list. 
When the nurses clicked the reminders, a window is displayed on the screen (Figure 4). We also provided the quick links when the nurses clicked the reminders (Figure 5). Nurses could click the nursing written assignments which they wanted to do right now. The nurses could complete the nursing written assignments in the time off from their workflow, instead of interrupting their workflow.

\begin{tabular}{|c|c|c|c|c|}
\hline \multicolumn{2}{|c|}{ 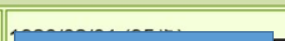 } & \multirow{2}{*}{ 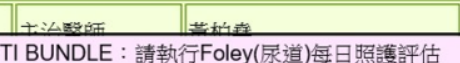 } & \multirow{2}{*}{\multicolumn{2}{|c|}{$4 \mathrm{~A} \& \mathrm{~B}$}} \\
\hline & & & & \\
\hline & & SI BUNDLE : 請執行CVC(腹股渾)每日照護評估 & 日期 & $2021 / 05 / 11 \ldots$ \\
\hline 跨領域 & & 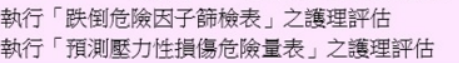 & & \\
\hline
\end{tabular}

Figure 4. The nurse clicked the reminders, it opened another window on the screen.

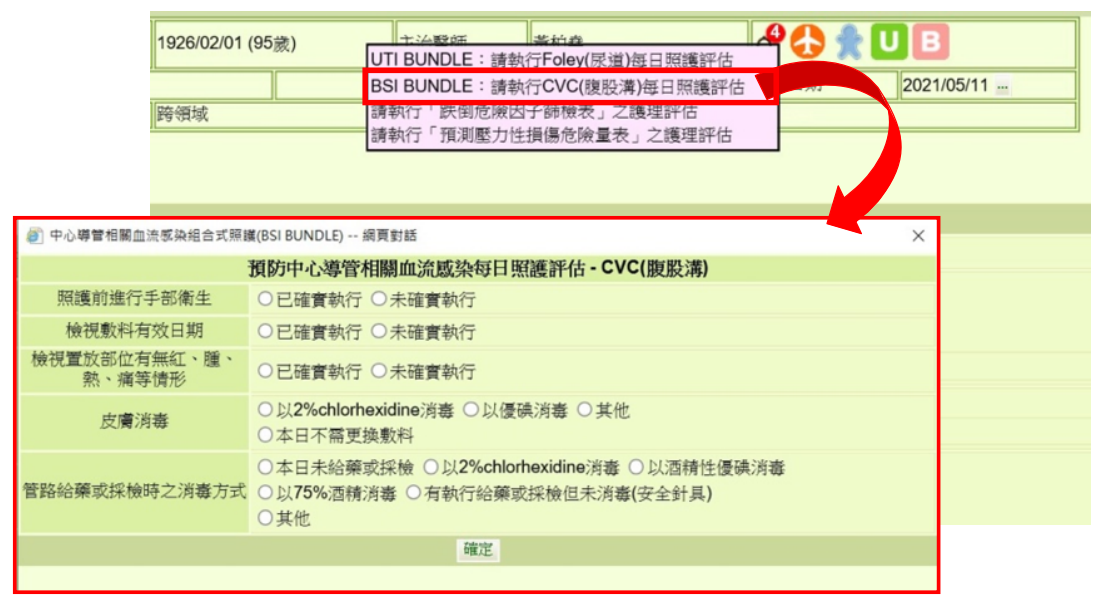

Figure 5. The quick link showed up when the nurse clicked the reminders.

\section{Results}

By adapting the "Integrated Reminders of Nursing Written Assignment", the average number of nursing written assignments raised from 3.42 to 3.86. The number of nursing interruptions of pop-up windows decreased from 489.48 to 0 in a shift. And the time of pop-up windows occupying the screen decreased from 3720.02 seconds to 0 in a shift. The completeness of the nursing written assignments raised from $97.3 \%$ to $100 \%$.

\begin{tabular}{ccccc}
\hline & $\begin{array}{c}\text { Nursing } \\
\text { written } \\
\text { assignment } \\
\text { (number of } \\
\text { times) }\end{array}$ & $\begin{array}{c}\text { Nursing } \\
\text { interruptions } \\
\text { (number of } \\
\text { times) }\end{array}$ & $\begin{array}{c}\text { Time of closing } \\
\text { pop-up } \\
\text { window } \\
\text { (second) }\end{array}$ & $\begin{array}{c}\text { The completeness of } \\
\text { nursing written } \\
\text { assignment }(\%)\end{array}$ \\
\hline Before & 3.42 & 489 & 3720 & $97.3 \%$ \\
After & 3.86 & .48 & 02 & $100 \%$ \\
\hline
\end{tabular}

Table 1. The evaluation of the "Integrated Reminders of Nursing Written Assignment". 


\section{Discussion}

With the advance of technology, nursing information system can simplify information storage and retrieval. However, with the rapid growth rate in healthcare data to be recorded, the traditional reminder (pop-up windows) may be not suitable for nurses nowadays. In order to suit the needs of the nurses, we adopted the most common reminders in social media reminders to the nursing information system. The nurses can complete the documents without interruptions of nursing workflow and require essential information when they need help.

\section{Conclusions}

In this study, we found that the "Integrated Reminders of Nursing Written Assignment" can effectively reduce the frequency of nursing interruptions, raise the completeness of the nursing written assignments. These designs are helpful to improve the quality of nursing care.

\section{Acknowledgements}

We would like to thank the Department of Nursing and the Department of Informatics for their valuable help in establishing the system.

\section{References}

[1] Sassaki RL, Cucolo DF, Perroca MG. Interruptions and nursing workload during medication administration process. Rev Bras Enferm.72 (2019), 1001-1006.

[2] Binobaid S, Almeziny M, Fan IS, Using an integrated information system to reduce interruptions and the number of non-relevant contacts in the inpatient pharmacy at tertiary hospital. Saudi Pharm J. 25(2017), 760-769. 